\title{
Bispecifics and CAR T cells that target intracellular cancer driver mutations Sandra Gabelli ${ }^{1}$, Katharine Wright ${ }^{2}$, Micheller Miller ${ }^{3}$, Emily Han-Chung Hsiue ${ }^{4}$, Michael S. Hwang ${ }^{5}$, Puchong Thirawatananond ${ }^{6}$, P. Aitana Azurmendi ${ }^{7}$, Jackie Douglass ${ }^{8}$, Tihitina Aytenfisu ${ }^{9}$, Michael Murphy ${ }^{10}$, Brian J Mog ${ }^{11}$, Alexander H. Pearlman ${ }^{12}$, Drew M. Pardoll ${ }^{13}$ \\ ${ }^{1}$ Johns Hopkins University ${ }^{2}$ No affiliation given, ${ }^{3}$ Johns Hopkins University, ${ }^{4}$ Sidney Kimmel Comprehensive Cancer Center, ${ }^{5}$ Sidney Kimmel Comprehensive Cancer Center, ${ }^{6}$ Johns Hopkins University, ${ }^{7}$ Department of Biophysics and Biophysical Chemistry, ${ }^{8} \mathrm{Johns}$ Hopkins University, 9 Johns Hopkins University School of Medicine, ${ }^{10} \mathrm{Cytiva},{ }^{11}$ Sidney Kimmel Comprehensive Cancer Center, ${ }^{12}$ Sidney Kimmel Comprehensive Cancer Center ${ }^{13}$ Sidney Kimmel Comprehensive Cancer Center gabelli@jhmi.edu
}

\begin{abstract}
The emergence of immunotherapy as an important tool in the fight against cancer takes advantage of the exquisite selectivity of antibodies. Until now successful targets, however, are limited to those on the cell surface, while most driver mutations occur in the genes encoding intracellular proteins. To overcome this limitation, antibodies can be engineered to target mutation derived neoantigens, peptides derived from mutant proteins that are presented on the cell surface by the Major Histocompatibility Complex Class I (MHC-I). In cases where both the wild-type (WT) and mutant peptide are presented, antibodies can be developed that selectively target the specific mutant peptide-HLA complex. We have selected antibody fragments by phage display that target mutant peptides derived from a tumour suppressor gene, specifically the R175H mutation in the protein p53 and from an oncogene (1), the R140Q mutation in isocitrate dehydrogenase 2 (IDH2).
\end{abstract}

The IDH2R140 and IDH2R140Q peptide-HLA:B*07 complexes revealed each peptide bound in an almost identical conformation with the mutant residue buried deep in the peptide-HLA-B*07 binding cleft. The structure of the antibody fragment, 2Q.1, in complex with the IDH2R140Q peptide-HLA:B*07 displayed a subtle backbone movement in the mutant peptide that underlies the observed antibody selectivity. Structure-guided optimization of the targeting moiety resulted in the development of a CAR T cell that lacks WT binding making it more selective and with improved on-target activity in response to IDH2R140Q-HLA-B*07, as assessed by IFN- $\gamma$ release. In contrast to the buried epitope in IDH2R140Q-HLA:B*07, the histidine residue in the $\mathrm{p} 53$ neoantigen-HLA:A*02 structure is exposed and available for antibody binding1. Structural basis of selectivity of the antibody against p53R175H, H2, is supported by a "cage-like" structure formed by tyrosine residues in three complementarity determining regions. Grafting of $\mathrm{H} 2$ anti $\mathrm{p} 53 \mathrm{R} 175 \mathrm{H}$ into a bispecific antibody with an anti CD3 fragment is effective in activating $\mathrm{T}$ cells to secrete cytokines and kill cancer cells. Moreover, treatment with $\mathrm{H} 2$ resulted in regression of human tumor xenografts in mice. Moreover, to investigate potential on-target off-tumour reactivity, the human peptidome was screened in silico for the recognition motif of the $\mathrm{H} 2$ antibody. Of the three possible candidate proteins identified, none showed evidence of triggering $\mathrm{T}$ cell activation via $\mathrm{H} 2$ bispecific in vitro.

The exquisite selectivity achievable with antibodies provides the added benefit of distinguishing between WT and mutant proteins-the foundation for developing effective treatments with minimal adverse effects to patients. While it remains to be determined which class of precision therapeutic will prove more efficacious in humans, we present here two avenues to treat cancer harboring public intracellular targets(2). The 2Q.1 CAR T cell approach allows for the incorporation of antigen dependent co-stimulation, a property that has proved essential in the successful translation of CAR T cells to the clinic. On the other hand, the $\mathrm{H} 2$ bispecific to treat $\mathrm{p} 53 \mathrm{R} 175 \mathrm{H}$ dependent cancers has the potential to be an "off-the-shelf" therapy, that does not require manipulation of patients own $\mathrm{T}$ cells and has a relatively simple manufacturing process.

1. Han-Chung Hsie E, Wright KM, Douglas J, Hwang MS, Mog BJ, Pearlman AH, Paul S, DiNapoli SR, Konig MF, Wang Q, Schaefer A, Miller MS, Skora AD, Azurmendi PA, Murphy MB, Liu Q, Watson E, Li Y, Pardoll DM, Bettegowda C, Papadopoulos N, Kinzler KW, Vogelstein B, Gabelli SB*, Zhou S. Targeting a neoantigen derived from a common TP53 mutation. Science. 2021 Mar 5;371(6533):eabc8697. doi: 10.1126/science.abc8697. Epub 2021 Mar 1. PMID: 33649166

2. Pearlman AH, Hwang MS, Konig MF, Han-Chung Hsie E, Douglass J, DiNapoli SR, Mog BJ, Bettegowda C, Pardoll DM, Gabelli SB, Papadopoulos N, Kinzler KW, Vogelstein B, Zhou S. Targeting public neoantigens for cancer immunotherapy. Nature Cancer volume 2, pages 487-497 (2021), 